\title{
AMOSTRAGEM PARA INVENTÁRIO FLORESTAL COM PROBABILIDADE DE SUPERPOSIÇÃO DE PARCELAS CIRCULARES ${ }^{1}$
}

\author{
Thomaz Corrêa de Castro da Costa² e Adair José Regazzi ${ }^{3}$
}

\begin{abstract}
RESUMO Este trabalho teve o propósito de avaliar uma metodologia de amostragem que propõe o uso de parcelas circulares superpostas em inventários florestais, comparada à amostragem simples ao acaso convencional. Compararam-se os métodos com parcelas retangular e circular de raio fixo e variável (Bitterlich). Os resultados mostraram que o método de parcelas circulares superpostas pode ser aplicado com os estimadores da amostragem simples ao acaso, e o método de Bitterlich pode ser uma alternativa à parcela circular de raio fixo.
\end{abstract}

Palavras-chave: Amostragem simples ao acaso, Amostragem por ponto e Método de Bitterlich.

\section{SAMPLING FOR FOREST INVENTORY WITH PROBABILITY OF CIRCULAR PLOT SUPERIMPOSING}

\begin{abstract}
The objective of this study was to evaluate a sampling methodology that proposes the use of overlapping circular plots in forest inventories in comparison with the conventional simple random sampling. The overlapping circular plots method was compared with rectangular and circular plots with fixed and variable radius (Bitterlich). Results showed that the ciclular plot superposing method may be applied with estimators of conventional simple random sampling, and the Bitterlich method may represent an alternative to the circular plot with fixed radius.
\end{abstract}

Keywords: Random simple sampling, Point sampling and Bitterlich's method.

\section{INTRODUÇÃO}

Em inventários realizados em florestas homogêneas é comum o uso de parcelas circulares como unidades de amostra (SOUZA, 1981), em que a locação é rápida e não fica sujeita à erros de dimensão como na marcação de parcelas retangulares (RIOS, 1993). No entanto, há uma pequena imperfeição conceitual, conforme o desenho amostral adotado. A população pode não ser totalmente coberta por $(\mathrm{N})$ parcelas circulares não superpostas, chegando a excluir até $21 \%$ da população (YANDLE e WIANT, 1981), que, dividida em N parcelas mutuamente exclusivas, não é exaustivamente coberta (Figura 1).

Fowler e Davis (1979) verificaram que essa situação pode afetar as estimativas em função da dimensão e distribuição espacial das árvores. Quando a distribuição das árvores é uniforme, as diferenças entre os parâmetros da subpopulação e da população se aproximam de zero.

No desenho sistemático, a certeza de excludência de áreas do povoamento é menos provável pelo fato de, na prática, a seleção de unidades de amostra ser definida pela distância, e, quando não ocorre diagramação prévia da população em unidades de amostra para o sorteio, qualquer região da população terá a mesma probabilidade de ser selecionada, e sendo essa imperfeição no entanto, contornada.

No caso de $\mathrm{N}$ parcelas circulares não superpostas, os estimadores da Amostragem Simples ao Acaso (ASA) são definidos para uma subpopulação e não para a população total, não permitindo a seleção da área excluída entre os círculos.

\footnotetext{
${ }^{1}$ Recebido em 08.01.2008 e aceito para publicação em 14.10.2009.

${ }^{2}$ Empresa Brasileira de Pesquisa Agropecuária, Embrapa, Brasil. E-mail: <thomaz@ cnpms.embrapa.br>.

${ }^{3}$ Departamento de Informática, Universidade Federal de Viçosa - Vicosa, MG - Brasil. E-mail: <adairreg@ufv.br>.
} 


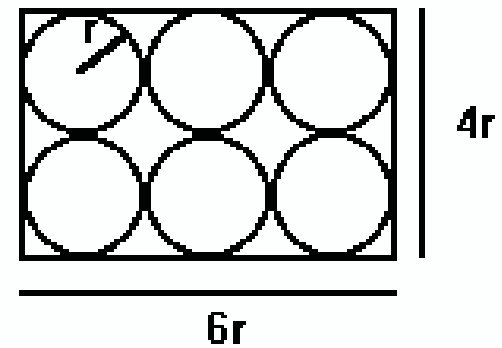

Figura 1 - População subdividida em parcelas circulares não superpostas de raio r.

Figura 1 - Population subdivided in nonsuperimposed Circular Plots with r radius.

Palley e O'Regan (1961), Schreuder (1970), Fowler e Davis (1979), Yandle (1979) e Yandle e Wiant (1981) abordaram essa questão focando um método de amostragem que permite a superposição de parcelas.

Neste trabalho foram discutidas conclusões obtidas pelos referidos autores, apresentado o método proposto por Yandle e Wiant (1981) que permite a superposição de parcelas circulares como aproximação do método de Bitterlich e verificadas as probabilidades de seleção de até duas árvores entre o método da Amostra Simples ao Acaso com Parcelas Retangulares (ASA) e o método da Amostra Simples ao Acaso com Superposição de Parcelas Circulares (ASAC). Por fim, procedeu-se à comparação entre os metodos de amostragem ao acaso e sistemático, com parcelas retangulares de raios fixo e variável, por meio de ensaios amostrais em um povoamento simulado, o que permitiu avaliar, na prática, o resultado com parcelas circulares de raios fixo e variável.

\subsection{Amostragem ao Acaso com Parcelas Circulares de Raio Fixo (ASAC)}

Um povoamento florestal pode ser amostrado com parcelas circulares permitindo a superposição de parcelas, como é feito na amostragem pelo método de Bitterlich ou amostragem por ponto (YANDLE, 1979).

Grosenbaugh (1952, 1958 citado por PALLEY e HORWITZ,1961) foi o primeiro a reconhecer amostragem por ponto (método de Biterlich) como um caso de amostragem com probabilidade proporcional ao tamanho (PPS) com reposição. Na amostragem por ponto, após definir a constante $\mathrm{k}$, proporção que determina o angulo de seleção da árvore qualificada, cada árvore terá a

R. Árvore, Viçosa-MG, v.34, n.1, p.137-145, 2010
Fonte: PALLEY e HORWITZ,1961.

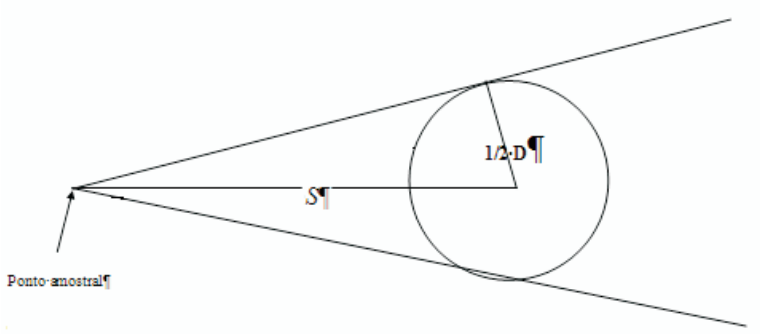

Figura 2 - Norma geométrica para amostragem por ponto. Figure 2-Geometric norm for point sampling.

probabilidade de ser selecionada proporcionalmente ao seu diâmetro $D$, que será dada por $\mathrm{P}=\pi S^{2} / A$, sendo $A$ a área amostrada e $\pi S^{2}$, a área do K- círculo da árvore (círculo com área proporcional à área basal da árvore), ou seu diâmetro, de acordo com o valor de K, que é dado por $K=\frac{S}{(1 / 2) D}$, sendo $S$ a distância entre o ponto selecionado e o centro da área basal da árvore qualificada; e $D$, o DAP da árvore qualificada (Figura 2).

Dessa forma, qualquer ponto na região $\pi S^{2} \mathrm{da}$ respeciva árvore que for selecionado estará incluindo a árvore. De acordo com a ilustração de uma população com três árvores (Figura 3), para amostragem por ponto uma unidade de amostra será definida a partir da seleção aleatória de um ponto na área da floresta (A). Toda árvore $\mathrm{i}$, em que a relação $\frac{S_{i}}{(1 / 2) D_{j}} \leq K$, estará incluída nessa unidade amostral. O conjunto dessas árvores irá fornecer um agrupamento associado àquele ponto amostral. Todo ponto selecionado dentro do Kcírculo da árvore 1 a incluirá. Da mesma forma, os pontos que forem selecionados dentro das áreas de interseção incluirão as árvores que tem seus K-círculos se superpondo formando essas áreas (Figura 3).

Yandle (1979) afirmou que a amostragem com parcelas de raio fixo, com locação aleatória de pontos (centros das parcelas), pode ser considerada como um caso especial da amostragem de Bitterlich, em que a probabilidade de seleção da árvore individual é constante, o que significa considerar todas as árvores com a mesma área basal. Pode-se utilizar, por exemplo, o diametro médio obtido pela amostra, Dm, sendo $K=\frac{S}{(1 / 2) D m}$, sendo $S$ o raio fixo da parcela. 
Fonte: Adaptado de PALLEY e O'REGAN,1961. Source: Adapted of the PALLEY e O'REGAN, 1961.
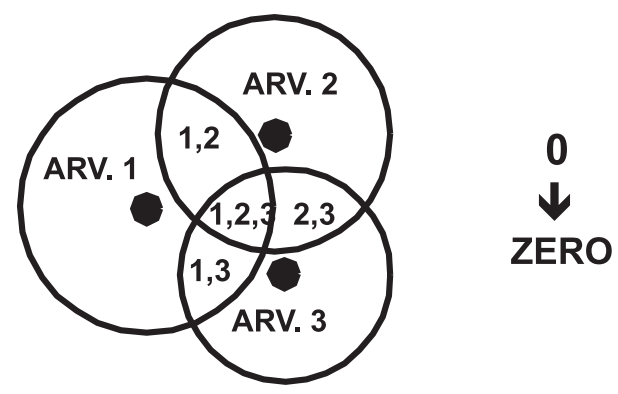

Figura 3 - População com agrupamentos de árvores e regiões definida para a amostragem por ponto, considerandose uma floresta com três árvores.

Figure 3 - Population with tree clusters and regions for point sampling considering a forest with three trees.

A relação entre a amostragem de Bitterlich e a amostragem com parcelas de raio fixo pode ser também obtida através da extensão do trabalho de Schreuder (1970), em que o agrupamento é uma coleção de pontos (áreas de interseção entre os k-círculos) contendo as mesmas árvores amostradas. Para adequar a população a uma grade finita de pontos, um agrupamento é definido como uma coleção de árvores amostradas associadas com um ponto, e diferentes pontos podem conter as mesmas árvores.

Com isso, Schreuder (1970) associou a amostragem por Bitterlich ao modelo de Amostragem Simples ao Acaso (ASA), restringindo a possibilidade de locação do centro da parcela a pontos de interseção entre linhas de uma grade. Considerando parcelas circulares de raio fixo, pode-se definir essa grade de pontos como uma população de $\mathrm{N}$ pontos (centros de parcelas de raio fixo), para a Amostragem Simples ao Acaso com Parcelas circulares Superpostas (ASAC), resultando na população (Figura 4).

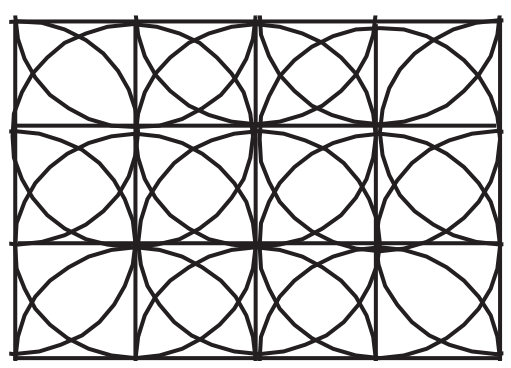

Figura 4 - População com parcelas circulares superpostas. Figure 4-Population with superimposed circular plots.
Schreuder (1970) mostrou que os estimadores da Amostragem Simples ao Acaso com Parcelas circulares Superpostas (ASAC), considerando o número de linhas da grade aproximadamente infinito, são os mesmos da amostragem de Bitterlich, derivados por Palley e Horwitz (1961 citados também por YANDLE e WIANT, 1981). Ao substituir parcelas de Bitterlich por parcelas de raio fixo nas fórmulas, todos os resultados se reduzem, no limite, para amostragem com parcelas de raio fixo (YANDLE, 1979).

Similarmente, no trabalho de Palley e O'Regan (1961) o segundo exemplo pode ser aplicado com a amostragem de parcelas de raio fixo, substituindo os círculos com áreas proporcionais às áreas basais das árvores (k-círculos) por círculos de mesma área.

Os estimadores para Amostragem Simples ao Acaso com Parcelas circulares superpostas de Raio Fixo (ASAC), não viesados, para o total e a variância do total, apresentados por Yandle (1979) e substanciados por Schreuder (1970) e Palley e O'Regan (1961), são:

$$
\begin{gathered}
\hat{Y}=\frac{A}{a}\left(\frac{1}{n} \sum_{i=1}^{n} T_{i}\right)=\frac{A}{a} \bar{T} \\
v(\hat{Y})=v\left(\frac{A}{a} \bar{T}\right)=\frac{A^{2}}{a^{2} n^{2}} v\left(T_{i}\right)=\frac{A^{2}}{a^{2}}\left[\frac{n \sum_{i=1}^{n} T_{i}^{2}-\left(\sum_{i=1}^{n} T_{i}\right)^{2}}{n^{2}(n-1)}\right]
\end{gathered}
$$

, sendo $n$ o número de parcelas ou pontos amostrados (centros selecionados das parcelas); $T_{i}$, o total da característica de interesse para a parcela i; $a$, a área da parcela; e $A$, a área da população amostrada.

Essas fórmulas são as mesmas da ASA, com reposição, considerando $N=\frac{A}{a}$, com a simplificação da fórmula para a variância do total sendo possível porque a seleção dos pontos é independente e, como as intensidades de amostra em inventários florestais são geralmente muito baixas $(<0,05)$, a probabilidade de ocorrer superposição de parcelas na amostra é muito pequena.

\subsection{Variâncias da ASAe ASAC}

Yandle e Wiant (1981) apresentaram as diferenças entre as variâncias dos estimadores para o total, obtidas pela ASA, com o uso de parcelas retangulares não superpostas; e ASAC, com parcelas circulares

R. Árvore, Viçosa-MG, v.34, n.1, p.137-145, 2010 
superpostas, em função dos elementos (árvores). A variância para ASA, ignorando a correção das populações finitas e considerando $N=\frac{A}{a}$ (dedução em Yandle e Wiant, 1981), é,

$V\left(\hat{Y}_{A S A}\right)=\frac{1}{n}\left[\frac{A}{a} \sum_{j=1}^{M} y_{j}^{2}-Y^{2}+\frac{A}{a} \sum_{i=1}^{N}\left(\sum_{\substack{\alpha=1 \\ m_{i}}}^{m_{i}} \sum_{\substack{\beta \\ \alpha \neq \beta}} y_{i \alpha} y_{i \beta}\right)\right]$

sendo $M$ o número total de árvores na população; $y_{j}, \mathrm{o}$ valor da característica de uma árvore j; $Y$, o valor total da característica; $N$, o número total de parcelas; $m_{i}$, o número total de árvores na parcela $\mathrm{i} ; y_{i \alpha}$ o valor da característica da árvore $\alpha$ na parcela i; $y_{i \beta}$, valor da característica da árvore $\beta$ na parcela i; e $n$, o número de parcelas amostradas.

Nota-se que o número de termos de produtos cruzados,

$+\frac{A}{a} \sum_{i=1}^{N} y_{i 1} y_{i 2}+y_{i 1} y_{i 3}+y_{i 2} y_{i 1}+y_{i 2} y_{i 3}+y_{i 3} y_{i 1}+y_{i 3} y_{i 2}$

(exemplo com 3 árvores na parcela), é dado pelo somatório dos arranjos de $\mathrm{m}_{\mathrm{i}}$ árvores duas a duas em cada parcela, $\sum_{i=1}^{N} m_{i}\left(m_{i}-1\right)$, sendo, $m_{i}\left(m_{i}-1\right)=A_{m_{i}}^{2}=\frac{m_{i} !}{\left(m_{i}-2\right) !}$, $A^{3}{ }_{2}=6$ produtos cruzados para o exemplo.

A variância da Amostragem ao Acaso com Parcelas Circulares de Raio Fixo (ASAC) é dada por Yandle (1979):

$V\left(\hat{Y}_{A S A C}\right)=\frac{1}{n}\left[\frac{A}{a} \sum_{j=1}^{M} y_{j}^{2}-Y^{2}+\frac{A}{a} \sum_{\alpha \neq}^{M} \sum_{\beta}^{M} y_{\alpha} y_{\beta}\left(\frac{a_{\alpha \beta}}{a}\right)\right]$ , sendo $y_{\alpha}$, o valor da característica da árvore $\alpha ; y_{\beta}$, o valor da característica da árvore $\beta ; a_{\alpha \beta}$, a área de superposição entre os círculos das árvores $\alpha$ e $\beta$; e os outros termos previamente definidos.

As variâncias diferem apenas nos termos de produtos cruzados. O número desses termos é o arranjo envolvendo todas as árvores do povoamento:

$$
A_{M}^{2}=M(M-1)
$$

Para ASAC, o número de termos de produtos cruzados irá depender do tamanho da parcela (a) e da densidade do povoamento, porque quaisquer árvores $\alpha$ e $\beta$ que estiverem a uma distância maior que o diâmetro da parcela não estarão incluídas na mesma parcela, resultando em $a_{\alpha \beta}=0$. Assim, o número de termos vai ser consideravelmente menor que $M(M-1)$.

R. Árvore, Viçosa-MG, v.34, n.1, p.137-145, 2010

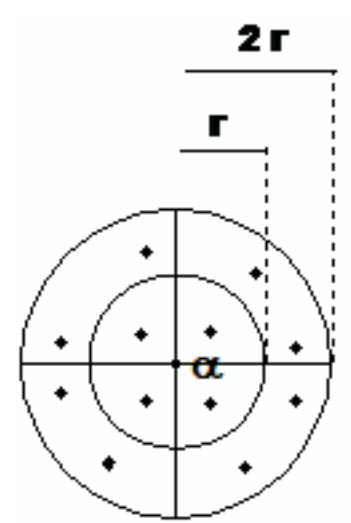

Figura 5 - Região de possíveis produtos cruzados com a árvore a.

Figure 5-Region of possible products crossed with the a tree.

A seguinte aproximação empírica é apresentada (YANDLE e WIANT, 1981), ou seja: considerando um tamanho de parcela com raio $r$, determinada árvore $\alpha$ centrada numa área de raio $2 \mathrm{r}$ vai ter $4 \mu-1$ grupos de árvores obtendo produto cruzado com $\alpha\left(a_{\alpha \beta}>0\right)$, sendo

$$
\mu=\frac{1}{N} \sum_{i=1}^{N} m_{i}
$$

o número médio de árvores por parcela e considerando uma distribuição das árvores razoavelmente regular na área (Figura 5).

Assim, o número de termos de produtos cruzados diferentes de zero dentro $V\left(\hat{Y}_{A S A C}\right)$ de será, aproximadamente, $\mathrm{M}(4 \mu-1)$.

Yandle e Wiant (1981) também calcularam o valor médio esperado da razão $\frac{a_{\alpha \beta}}{a}$, chegando ao seguinte resultado: $E\left(\frac{a_{\alpha \beta}}{a}\right)=\frac{1}{4}$. Combinando esse resultado com o número esperado de termos de produtos cruzados dentro da $V\left(\hat{Y}_{A S A C}\right)$, é apresentada a contribuição relativa desses termos: $\frac{1}{4} M(4 \mu-1)=M\left(\mu-\frac{1}{4}\right)$.

Para ASA, em populações com espaçamento fixo entre as árvores, sendo $\mathrm{M}$ uma constante, o número médio esperado é:

$E\left[\sum_{i=1}^{N} m_{i}\left(m_{i}-1\right)\right]=\sum_{i=1}^{N} E\left(m_{i}^{2}-m_{i}\right)=\sum_{i=1}^{N} \mu(\mu-1)=N \mu(\mu-1)=M(\mu-1)$

Este valor será menor que o correspondente para ASAC, com valores de variancia menores para amostragem sem superposição de parcelas, 
considerando-se a mesma população amostrada, a intensidade de amostragem e o tamanho da parcela.

\section{MATERIAL E MÉTODOS}

A dedução das probabilidades de seleção de amostras e árvores na Amostragem Simples ao Acaso com Parcelas Retangulares (ASA) e com parcelas circulares superpostas (ASAC) foi executada para obter a comparação entre ambas. O desenvolvimento foi a partir de algumas expressões com deduções não apresentadas no artigo de Yandle e Wiant (1981).

Foi gerada uma população fictícia para execução de ensaios amostrais comparando ASA, ASAC, método de Bitterlich e distribuição sistemática de unidades de amostra. A avaliação das estimativas da população de 75 árvores distribuídas aleatoriamente em 1 ha, com o parâmetro área seccional, foi obtida pela exatidão com o parâmetro populacional e pelo tamanho da variancia do total. Para essa população foram estabelecidos os seguintes desenhos e ensaios amostrais:

1. Dezesseis parcelas retangulares de $25 \times 25 \mathrm{~m} \mathrm{e}$ circulares não superpostas de raio 12,5 m (Figura 6), obtendo-se 200 amostras aleatórias de quatro parcelas para cada desenho (parcelas retangulares e circulares).

2. Grade de 64 pontos centrais de parcelas circulares de raio fixo 12,5 m, superpostas (Figura 7), obtendo-se 200 amostras aleatórias de quatro parcelas.

3. Uma amostra sistemática de quatro pontos pelo método de Bitterlich.

4. Uma amostra sistemática de quatro parcelas ciculares de raio fixo $12,5 \mathrm{~m}$.

5. Três amostras aleatórias de quatro pontos pelo método de Bitterlich.

6. Três amostras aleatórias de quatro parcelas circulares de raio fixo $12,5 \mathrm{~m}$.

A população, os desenhos e os ensaios amostrais foram realizados a partir de dados reais de árvores, com procedimentos em sistemas de informações geográficas (EASTMAN, 2003) e linguagem Visual Basic em Excel.

\section{RESULTADOS E DISCUSSÃO}

\subsection{Probabilidades de Seleção para ASA e ASAC}

Comparando a Amostragem Simples ao Acaso com Parcelas Retangulares (ASA), com a Amostragem ao Acaso com Parcelas Circulares Superpostas (ASAC), é mostrada a diferença entre as probabilidades amostrais, considerando-se a seleção de até duas unidades de amostra.

\subsubsection{Probabilidade de seleção para ASA, com reposição}

A probabilidade de uma árvore a, presente em uma unidade de amostra, ser incluída na amostra é:

$\mathrm{P}_{\mathrm{r}}(\alpha)=\frac{N^{n}-(N-1)^{n}}{N^{n}} 1-(1-P)^{\mathrm{n}}$, sendo $N^{n}$ o número de todas as possíveis amostras; $(N-1)^{n}$, o número de todas as possíveis amostras sem a parcela que inclui a árvore $\alpha ; N^{n}-(N-1)^{n}$, o número de todas as possíveis amostras com a parcela que inclui a árvore $\alpha$; e $P=\frac{1}{N}=\frac{a}{\dot{A}}$ Uma vez que cada árvore está presente em uma e somente uma parcela, a probabilidade de a árvore a estar incluída na amostra será $1-(1-P)^{\mathrm{n}}$.

A probabilidade de duas árvores $\alpha$ e $\beta$ de parcelas distintas estarem na mesma amostra é:

$$
\mathrm{P}_{\mathrm{r}}(\alpha \bigcap \beta)=1-2(1-P)^{n}+(1-2 P)^{n} \text {, sendo } P=\frac{1}{N}=\frac{a}{A}
$$

Apresentando a dedução:

$$
\begin{aligned}
& P_{r}(\alpha \cap \beta)=1-P_{r}(\overline{\alpha \cap \beta})=1-P_{r}(\bar{\alpha} \cup \bar{\beta}) \\
& \quad=1-\left[P_{r}(\bar{\alpha})+P_{r}(\bar{\beta})-P_{r}(\bar{\alpha} \cap \bar{\beta})\right]
\end{aligned}
$$

Mas $P_{r}(\bar{\alpha} \cap \bar{\beta})=P_{r}(\overline{\alpha \cup \beta})$ representa a probabilidade de as árvores $\alpha$ e $\beta$ não ocorrerem juntas em nenhuma das $n$ parcelas da amostra, ou seja:

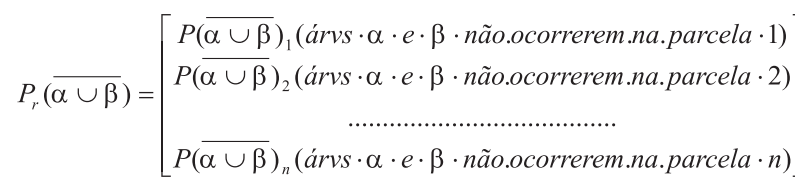

Como as $n$ parcelas são distribuídas aleatoriamente, $P_{r}(\overline{\alpha \cup \beta})=[P(\overline{\alpha \cup \beta})]^{n}=[1-P(\alpha \cup \beta)]^{n}$

$=\left\{1-[P(\alpha)+P(\beta)-P(\alpha \cap \beta)\}^{n}=[1-P(\alpha)-P(\beta)]^{n}\right.$ e sabendo que $P(\alpha)=P(\beta)=P$ e $\mathrm{P}_{\mathrm{r}}(\alpha)=\mathrm{P}_{\mathrm{r}}(\bar{\beta})=(1-P)^{\mathrm{n}}$, chega-se a probabilidade de inclusão de duas arvores em parcelas distintas para $n$ sorteios aleatórios com reposição:

$$
P_{r}(\alpha \cap \beta)=1-2(1-P)^{n}+(1-2 P)^{n}
$$

R. Árvore, Viçosa-MG, v.34, n.1, p.137-145, 2010 


\subsubsection{Probabilidade de seleção para ASAC}

A probabilidade de uma árvore a estar na amostra é $\mathrm{P}_{\mathrm{r}}($ árv. $\alpha)=1-(1-P)^{n}$. Apresentando a dedução, a probabilidade de a árvore $\alpha$ estar na amostra pode ser dada por:

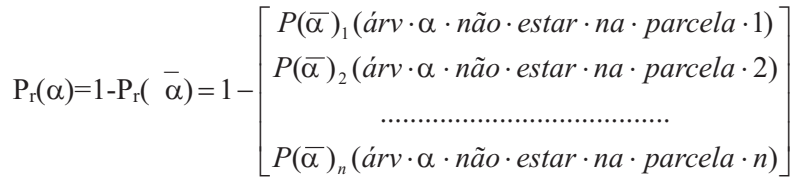

$\operatorname{Mas} P(\bar{\alpha})_{\mathrm{i}}$ (árv. $\alpha$ não estar em qualquer parcela $)=1-P$, sendo $P$ a probabilidade de a árvore $\alpha$ estar em qualquer parcela.

Como a seleção de pontos é independente, com $\mathrm{n}$ pontos escolhidos aleatoriamente, para que a árvore $\alpha$ não esteja em nenhuma das n parcelas, $\mathrm{P}_{\mathrm{r}}(\bar{\alpha})=$ $(1-P)(1-P) \ldots(1-P)=(1-P)^{\mathrm{n}}$.

Assim, a probabilidade de a árvore a estar na amostra será $\mathrm{P}_{\mathrm{r}}(\alpha)=1-(1-P)^{\mathrm{n}}$.

A probabilidade de duas árvores estarem ambas na amostra é dada por:

$\mathrm{P}_{\mathrm{r}}(\alpha \cap \beta)=1-2(1-P)^{n}+\left(1-2 P+P_{\alpha \beta}\right)^{n}, \operatorname{com} P_{\alpha \beta}=\frac{a_{\alpha \beta}}{A}$, sendo $a_{\alpha \beta}$, a área de superposição entre os círculos das árvores $\alpha$ e $\beta$.

Para deduzir a probabilidade de duas árvores estarem simultaneamente na amostra, obtém-se:

$$
\begin{gathered}
P_{r}(\alpha \cap \beta)=1-P_{r}(\overline{\alpha \cap \beta})=1-P_{r}(\bar{\alpha} \cup \bar{\beta}) \\
\quad=1-\left[P_{r}(\bar{\alpha})+P_{r}(\bar{\beta})-P_{r}(\bar{\alpha} \cap \bar{\beta})\right]
\end{gathered}
$$

$\operatorname{Mas} \operatorname{Pr}(\bar{\alpha} \cap \bar{\beta})=[P(\overline{\alpha \cup \beta})]^{n}=[1-P(\alpha \cup \beta)]^{n}$, $=\left\{1-[P(\alpha)+P(\beta)-P(\alpha \cap \beta)\}^{n}\right.$ conforme dedução para ASA. E, sabendo que $P(\alpha)=P(\beta)=P$ e, $P_{r}(\bar{\alpha})=P_{r}(\bar{\beta})=(1-P)^{n} \quad$ chega-se à fórmula da probabilidade de incluir duas árvores para n sorteios aleatórios com reposição, $P_{r}(\alpha \cap \beta)=1-2(1-P)^{n}+\left(1-2 P+P_{\alpha \beta}\right)^{n}$ como descrito em Yandle e Wiant (1981).

Nota-se que, na Amostragem Simples ao Acaso (ASA) com reposição, a probabilidade de dada árvore estar sendo incluída na amostra é a mesma de quando se usa ASAC. Já a probabilidade de a amostra incluir duas árvores consecutivas com parcelas não superpostas será também dada pela fórmula:

$$
P_{r}(\alpha \cap \beta)=1-2(1-P)^{n}+\left(1-2 P+P_{\alpha \beta}\right)^{n}
$$

Nota-se que o único termo que diverge em relação à probabilidade de inclusão de até duas árvores na Amostragem Simples ao Acaso (ASA) com reposição é $P_{\alpha \beta}$ o que, para ASAC, o valor de $P_{\alpha \beta}$ dependerá da proximidade entre as árvores $\alpha$ e $\beta$ e da área de intersecção das parcelas circulares.

\subsection{Desenhos e Ensaios Amostrais}

Nas Figuras 6 e 7 são apresentados os desenhos de amostragem no povoamento fictício com 75 árvores. As distribuições dos ensaios de maior controle, 1 e 2, com a seleção de 200 amostras para estabilização da estimativa (COSTA et al., 1996), são visualizadas nas Figuras 8,9 e 10. As amostras com parcelas circulares não superpostas, com exclusão de 12 árvores (16\%), e as amostras com parcelas superpostas apresentaram maior variabilidade nas estimativas.

Na comparação dos eventos amostrais, (Tabela 1) entre os ensaios com 200 repetições, a menor exatidão foi verificada no evento com parcelas circulares não superpostas, sendo o evento com parcelas retangulares o que mais se aproximou do parâmetro da população, obtendo também a menor variação da área basal total (s).

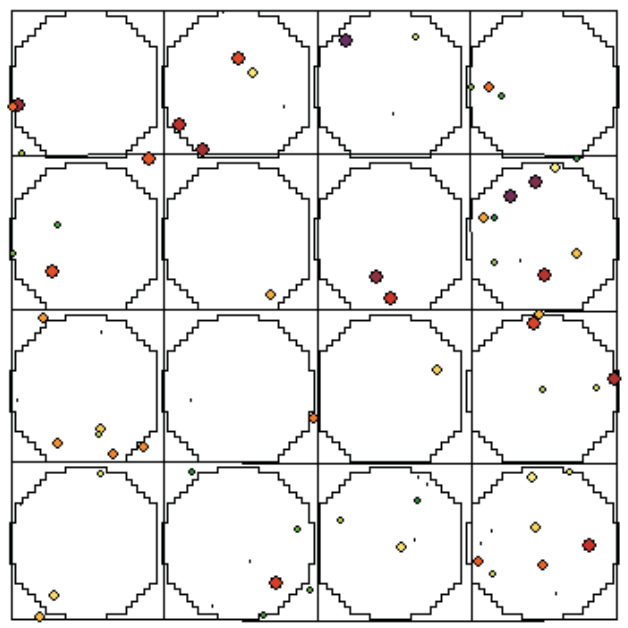

Figura 6 - Distribuição aleatória de 75 árvores no povoamento de 1 ha $(100 \times 100 \mathrm{~m})$, delimitado por 16 parcelas retangulares de $25 \times 25 \mathrm{~m}$ e 16 parcelas circulares de raio fixo de $12,5 \mathrm{~m}$.

Figure 6-Random distribution of 75 trees in 1 ha forest (100 x 100 meters) enclosed by 16 square plots of $25 \times 25$ meters and 16 circular plots of fixed radius of 12.5 meters. 
Nos ensaios sem repetição, as menores exatidões foram obtidas pelo procedimento sistemático, sem grandes diferenças entre o método de Biterlich e o de parcelas circulares de raio fixo. Outro estudo, conduzido por Farias et al. (2002), gerou resultados com maiores erros amostrais para o método de Bitterlich, aumentando sistematicamente com o fator de área basal (k).

Uma confirmação teórica desses ensaios é que uma população aleatória não deve ser inventariada pelo procedimento sistemático, seja por parcelas retangulares ou circulares. Embora esse processo seja mais viável operacionalmente, ele induz a perda de representatividade, por adotar um desenho uniforme em populações de natureza não uniforme.

A realização de inventário florestal considera, entre outros, o tamanho, a forma e a distribuição de unidades de amostra, e o objetivo de obter estimativas confiáveis e precisas dos parâmetros não deve ser negligenciado. No caso deste artigo, o modelo proposto por Yandle e Wiant (1981), embora de melhor precisão em relação ao procedimento de parcelas circulares não superpostas, não representa vantagem operacional em relação aos ensaios de pontos aleatórios. E o procedimento de Bitterlich, método não usual em inventários florestais, mostrou-se eficiente para a estimativa do parâmetro populacional.

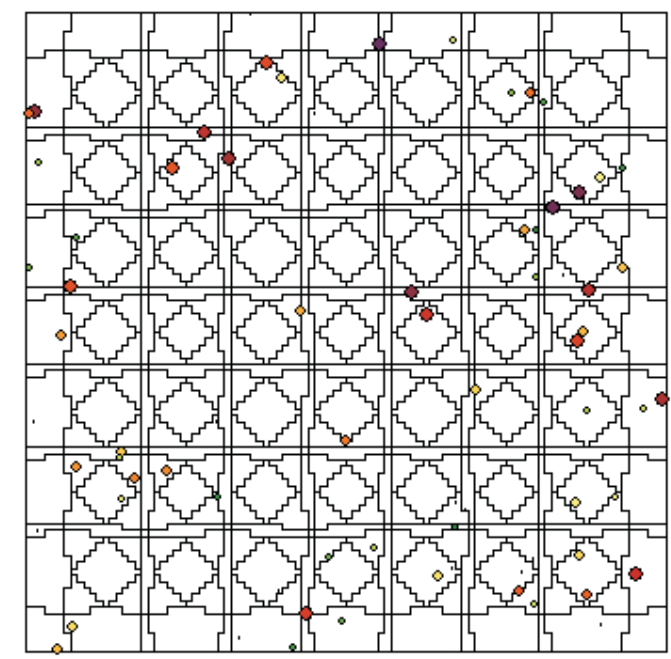

Figura 7 - Distribuição aleatória de 75 árvores no povoamento de 1 ha $(100 \times 100 \mathrm{~m})$, delimitado por 64 parcelas circulares superpostas com raio de $12,5 \mathrm{~m}$.

Figure 7 - Random distribution of 75 trees in 1 ha forest (100 100 meters) enclosed by 64 overlapping circular plots of $25 \times 25$ meters and 16 circular plots of fixed radius of 12.5 meters.

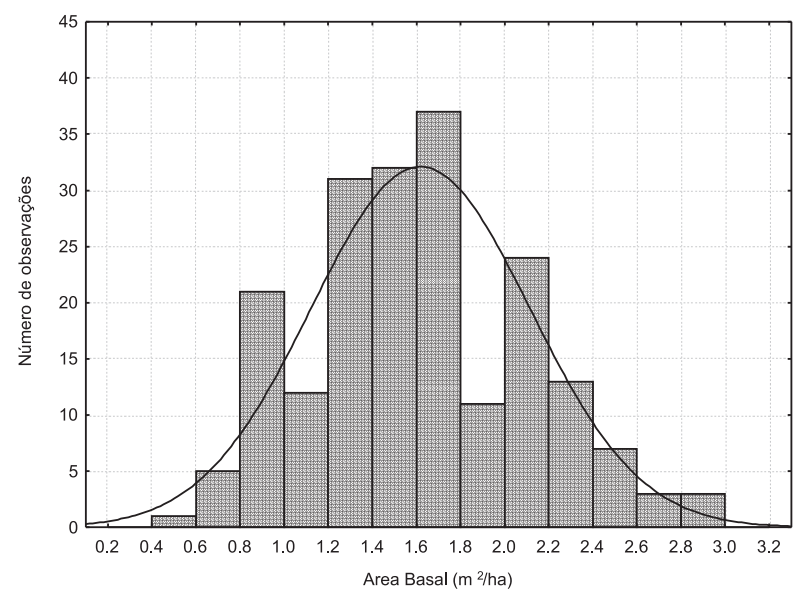

Figura 8 - Frequência de área basal $\left(\mathrm{m}^{2} / \mathrm{ha}\right)$ estimada de 200 amostras de quatro parcelas extraídas aleatoriamente da grade de 16 parcelas retangulares de $25 \times 25$ m. Distribuição Normal por teste Qui-Quadrado $\mathrm{t}=24,63952, \mathrm{gl}=7$ (ajustado) e, $\mathrm{p}=0,00088$.

Figure 8 - Frequency of the basal area $\left(\mathrm{m}^{2} / \mathrm{ha}\right)$ estimated by 200 samples from the 4 plots randomly extracted from the grid of 16 square plots of $25 \times 25$ meters. Normal function of chi-square test $t=24.63952$, $d f=7$ (adjusted) and $p=0.00088$.

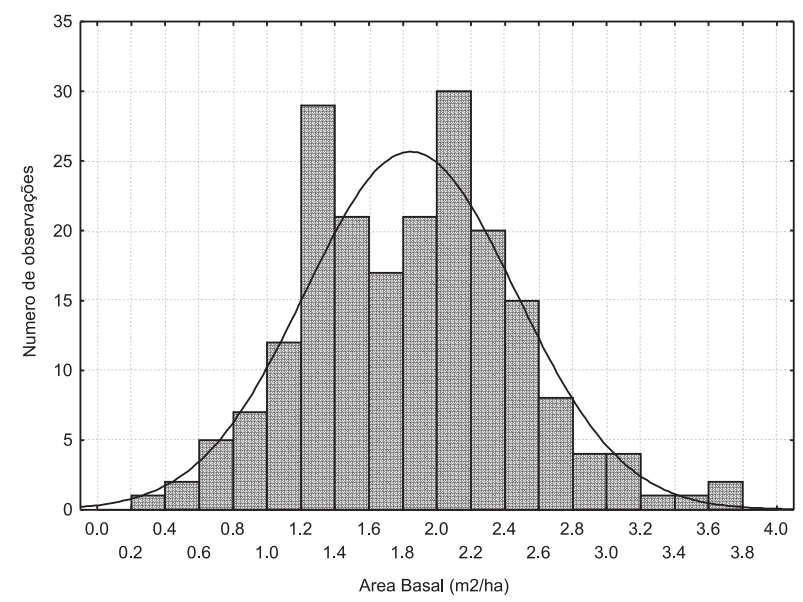

Figura 9 - Frequência de área basal $\left(\mathrm{m}^{2} /\right.$ ha) estimada de 200 amostras de quatro parcelas extraídas aleatoriamente da grade de 16 parcelas circulares não superpostas de raio de $12.5 \mathrm{~m}$. Distribuição normal por teste Qui-Quadrado $\mathrm{t}=14.68972, \mathrm{gl}=10$ (ajustado) $\mathrm{e} \mathrm{p}=0,14379$.

Figure 9 - Frequency of the basal area $(\mathrm{m} 2 / \mathrm{ha})$ estimated by 200 samples from the 4 plots randomly extracted from the grid of 16 non-overlapping circular plots of radius 12.5 meters. Normal function of chisquare test $t=14.68972, d f=10$ (adjusted) and $p=0.14379$.

R. Árvore, Viçosa-MG, v.34, n.1, p.137-145, 2010 
Tabela 1 - Parâmetro da população e estimativas de área basal $\left(\mathrm{m}^{2} / \mathrm{ha}\right)$ total e desvio-padrão do total, em diferentes ensaios amostrais.

Table 1 - Population parameter and estimates of the total basal área $\left(\mathrm{m}^{2} / \mathrm{ha}\right)$ and standard deviation of the total in different sampling assays.

\begin{tabular}{|c|c|c|c|c|}
\hline & Amostras & $\mathrm{Ab}\left(\mathrm{m}^{2} / \mathrm{ha}\right)$ & $\mathrm{S}\left(\mathrm{Abm}^{2} / \mathrm{ha}\right)$ & $\begin{array}{l}\mathrm{E}\left(\mathrm{Ab}^{\wedge} \mathrm{m}^{2} / \mathrm{ha}\right. \\
\left.-\mathrm{Abm} \mathrm{m}^{2} / \mathrm{ha}\right)\end{array}$ \\
\hline População & & 1,64 & & \\
\hline Retang_16 parcelas (200 amostras/4 parcelas) & 200 & 1,62 & 0,4971 & $-0,02$ \\
\hline CircRFix_16 parcelas (200 amostras/4 parcelas) & 200 & 1,84 & 0,6216 & 0,20 \\
\hline CircRFix_64 pontos (200 amostas/4 parcelas) & 200 & 1,71 & 0,6150 & 0,07 \\
\hline Biterlich ( $(\mathrm{k}=1)$ _Sistem & 1 & 2,75 & 0,5774 & 1,11 \\
\hline CircRFix_Sistem & 1 & 2,46 & 0,6965 & 0,82 \\
\hline Biterlich_Aleat1 & 1 & 1,66 & 1,0787 & 0,02 \\
\hline Biterlich_Aleat2 & 1 & 1,11 & 0,6168 & $-0,53$ \\
\hline Biterlich_Aleat3 & 1 & 1,30 & 0,5638 & $-0,34$ \\
\hline CircRFix_Aleat 1 & 1 & 1,68 & 1,0040 & 0,04 \\
\hline CircRFix_Aleat2 & 1 & 1,23 & 0,6601 & $-0,41$ \\
\hline CircRFix_Aleat3 & 1 & 1,33 & 0,7905 & $-0,31$ \\
\hline
\end{tabular}

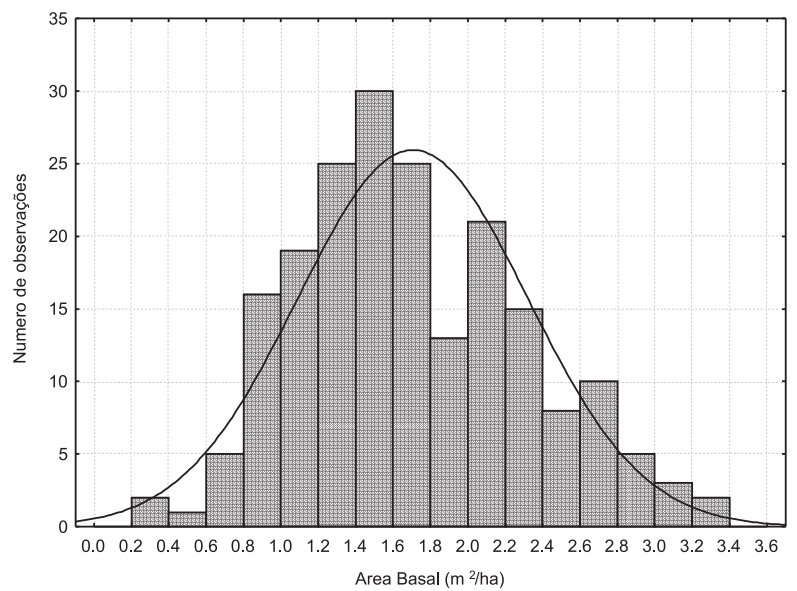

Figura 10-Frequência de área basal ( $\mathrm{m}^{2} / \mathrm{ha}$ ) estimada de 200 amostras de quatro parcelas extraídas aleatoriamente da grade de 64 parcelas circulares superpostas de raio de $12,5 \mathrm{~m}$. Distribuição normal por teste QuiQuadrado $\mathrm{t}=16,46096, \mathrm{gl}=10$ (ajustado) e $\mathrm{p}$ $=0,08718$.

Figure 10-Frequency of basal area $\left(\mathrm{m}^{2} / \mathrm{ha}\right)$ estimated by 200 samples from the 4 plots randomly extracted from the grid of 64 overlapping circular plots of 12.5 meters. Normal function of chi-square test $t=16.46096, d f=10$ (adjusted) and $p=$ 0.08718 .

\section{CONCLUSÕES}

Concluiu-se que, em levantamentos por amostragem em que o lançamento de unidades de amostra é feito aleatoriamente, sem considerar a diagramação de parcelas em mapas, o uso de parcelas circulares não provoca tendenciosidade nem imprecisão nas estimativas.

O método da ASAC aproxima-se do método da ASA, com parcelas retangulares, em termos de variância do total e probabilidade de seleção das unidades de amostra. E, como a intensidade de amostragem em inventários florestais é geralmente muito baixa $(<0,05)$, a probabilidade de superposição de parcelas é muito pequena. As fórmulas dos estimadores serão as usuais da ASA, podendo-se sortear aleatoriamente pontos em vez de parcelas na população e aplicar o método de Bitterlich como alternativa para a parcela circular de raio fixo, pelo seu bom desempenho nos resultados.

\section{REFERÊNCIAS}

EASTMAN, J. R. IDRISI Kilimanjaro guide to GIS and image processing. USA Manual Version 14.00. Worcester: Clark Labs, Clark University, 2003. 306p.

FARIAS, C. A. et al. Comparação de métodos de amostragem para análise estrutural de florestas ineqüiâneas. Revista Árvore, v.26, n.5, p.541$548,2002$.

FOWLER, G. W.; DAVIS C. F. Sampling natural resource populations: mutuallly exclusive fixedárea sampling units. Resource Inventory Notes, BLM-23: p.1-7,1979. 
PALLEY, M. N.; HORWITZ, L. G. Properties of some randon and Systematic point sampling estimators. Forest Science, v.7, n.1, p.52-65, 1961.

PALLEY, M. N.; O'REGAN, W. G. A Computer technique for the study of forest sampling methods. Foresty Science, v.7, n.1, p.282-294, 1961.

RIOS, N. A. Amostragem com igual probabilidade de seleção e amostragem com probabilidade proporcional ao tamanho, em plantações de Eucaliptos, Minas Gerais. 1993. 154f. Dissertação (Mestrado em Ciência Florestal) - Universidade Federal de Viçosa, Viçosa, MG, 1993.

SCHEREUDER, H. T. Point sampling theory in the framework of equal-probability cluster sampling. Foresty Science, v.16, n.2, p.240-246, 1970.
SOUZA, A. L. Comparação de tipos de amostragens, com parcelas circulares de área fixa e variável, em povoamentos de Eucalyptus grandis de origem híbrida, cultivados na região de Bom Despacho, Minas Gerais. 1981. 79f. Dissertação (Mestrado) - Universidade Federal de Viçosa,Viçosa, MG, 1981.

YANDLE, D. O. \& WIANT, H. V. Comparison of fixed radius circular plot sampling with simple random sampling. Forest Science, v.27, n.2, p.245-252, 1981 .

YANDLE, D. O. A generalization of techniques for sampling populations that are spatially distributed in two or more dimensions. Fort Collins: Forest Resources Inventories, 1979. p.966-982. 
